\title{
A systematic numerical study of the tidal instability in a rotating triaxial ellipsoid
}

\author{
D. Cébron ${ }^{\mathrm{a}, *}$, M. Le Bars ${ }^{\mathrm{a}}$, J. Leontini ${ }^{\mathrm{b}}$, P. Maubert ${ }^{\mathrm{a}}$, P. Le Gal ${ }^{\mathrm{a}}$ \\ ${ }^{a}$ Institut de Recherche sur les Phénomènes Hors Equilibre, UMR 6594, CNRS et Aix-Marseille Universités, 49 rue F. Joliot-Curie, BP146, 13384 \\ Marseille, France. \\ ${ }^{b}$ Department of Mechanical and Aerospace Engineering, Monash University, Clayton Campus Victoria 3800, Australia
}

\begin{abstract}
The full non-linear evolution of the tidal instability is studied numerically in an ellipsoidal fluid domain relevant for planetary cores applications. Our numerical model, based on a finite element method, is first validated by reproducing some known analytical results. This model is then used to address open questions that were up to now inaccessible using theoretical and experimental approaches. Growth rates and mode selection of the instability are systematically studied as a function of the aspect ratio of the ellipsoid and as a function of the inclination of the rotation axis compared to the deformation plane. We also quantify the saturation amplitude of the flow driven by the instability and calculate the viscous dissipation that it causes. This tidal dissipation can be of major importance for some geophysical situations and we thus derive general scaling laws which are applied to typical planetary cores.
\end{abstract}

Keywords: tides, tidal/elliptical instability, planetary cores, finite element numerical simulations

\section{Introduction}

Tides are large scale deformations induced by gravitational interactions that affect all the layers of a given planet or star. They play an important role in geo- and astrophysics and so, many studies are devoted to this subject. Their most obvious phenomena are of course the oceanic flows on Earth, but tides are also responsible for the intense volcanism on Io for instance. In stars but also in liquid planetary cores, tidal forcing induces an elliptical deformation of the rotating streamlines that may excite a parametric resonance of inertial waves called the elliptical or tidal instability. This instability could, for instance, be responsible for the surprising magnetic field in Io [23, 26, 13] and for fluctuations in the Earth's magnetic field on a typical timescale of 10,000 years [1]. It may also have a significant influence on the orbital evolution of binary stars [51] and moon-planet systems [31].

As described in the review of [22], the elliptical instability is a three-dimensional instability which may grow as soon as a rotating fluid possesses elliptical streamlines. As previously stated, it is due to a triadic parametric resonance between two inertial waves of the rotating fluid [24] and the underlying strain field responsible for the elliptic deformation [2, 61]. Such an instability has been found in many different contexts, including those where the strain field is due to vortex interactions or to elliptically deformed boundaries. It has thus been the focus of numerous theoretical and experimental studies, devoted to the dynamics of two-dimensional turbulent flows (e.g. [62, 45] or [17] for a viscoelastic fluid), to the stability of wakes [37], to the dynamics of vortex pairs [38, 36, 43], and to the flow inside deformed rotating cylinders [16, 41, 10, 11], ellipsoids [14, 21, 27] and shells [1, 53, 28]. More recently, magnetohydrodynamical effects of the elliptical instability have been investigated, with applications in MHD turbulence [58] or in induction processes in planets [26, 13].

From a numerical point of view, most studies have been devoted to the dynamics of deformed two-dimensional vortices in three-dimensional domains [e.g. 39, 56, 29, 52]. For instance, the seminal paper of [48] considered the growth of a perturbation on the planar velocity field associated with an elliptical vortex inside a box with zero normalflow boundary conditions, assuming periodicity in the axial direction; the linear eigenvalue problem was then solved

\footnotetext{
*corresponding author

Email address: cebron@irphe.univ-mrs.fr (D. Cébron)
} 
by spectral methods, and the growth rate of the elliptical instability was found. Numerical studies in rotating containers are less numerous. [42] used a non-orthogonal elliptico-polar coordinate system in order to solve the flow in an elliptically deformed cylinder by spectral methods; the non-linear temporal evolution of two different modes of the elliptical instability was calculated with no-slip boundary conditions on the sidewalls and stress-free conditions on the top and the bottom of the cylinder. [54] determined numerically the frequencies and the growth rates of the instability in both ellipsoid and ellipsoidal shell geometries using a linear Galerkin method, projecting the flow on a selected number of inertial waves. Finally, [55] studied the stability of self-gravitating compressible ellipsoidal fluid configurations and pointed out the occurence of the elliptical instability. However, to the best of our knowledge, the full non-linear evolution of the tidal instability in an ellipsoidal geometry has not yet been simulated numerically. This is the purpose of the present work, which aims at completing our previous theoretical and experimental investigations [27, 26, 13, 31] in accessing global quantities of fundamental importance for planetary applications.

In this paper, we focus on hydrodynamics only, leaving thermal and magnetic field interactions with the tidal instability for further investigations [e.g. 6]. In section 2, the system and the numerical method are presented. The numerical model is validated by comparison with the theoretical results found in the literature, regarding the flow in a rotating sphere submitted to a small and fixed elliptical deformation. In section 3 , we then study the influence of three additional complications of fundamental importance for geophysical applications: (i) the influence of the length of the ellipsoid along its rotation axis compared to the mean equatorial radius (i.e. the body oblateness in geophysical terms), (ii) the influence of a background rotation, corresponding to the orbital motion of the companion body responsible for the tidal deformation, and (iii) the influence of the inclination of the rotation axis compared to the deformation plane (i.e. the obliquity). In section 4 we quantify systematically two large-scale quantities relevant for planetary applications, namely (i) the amplitude of the instability at saturation, and (ii) the power dissipated by the instability. General laws in terms of dimensionless numbers are derived and applied to typical planetary cores in section 5

\section{Numerical model and its validation}

\subsection{Definition of the system and description of the numerical method}

The present study takes place in direct continuity of our experimental studies of the elliptical instability in a deformed spheroid [27, 26, 13, 31]. In these experiments, a hollow sphere of radius R, molded in a silicone cylinder, is filled with liquid and set in rotation at a constant angular velocity $\Omega$ about its axis $(O z)$, while it is slightly compressed by a quantity $s$ along the axis $(O x)$, perpendicular to the rotation axis. The geometry is then a triaxial ellipsoid of axes $(a, b, c)=(R-s, R+s, R)$, with an equatorial ellipticity $\varepsilon=\left(b^{2}-a^{2}\right) /\left(a^{2}+b^{2}\right)$ and a constant tangential velocity along the deformed boundaries, equal to $\Omega R$ at the equator. Such a configuration is a model for a liquid planetary core with no solid inner core (e.g. like the jovian moon Io or the early Earth), surrounded by a deformable mantle with a constant tangential velocity. Similarly, our numerical model studies the rotating flow inside an ellipsoid of axes $(a, b, c)$ related to the frame $(O x, O y, O z)$, with a constant tangential velocity all along the boundary in each plane perpendicular to the rotation vector $\mathbf{\Omega}$ (see the sketch in figure1). In order to extend our previous experimental study, the length $c$ of the ellipsoid can be chosen independently of the other lengths $a$ and $b$ (with $b>a$ ). Moreover, the rotation axis of the ellipsoid can be inclined compared to the c-axis. Note however that in this paper, unless otherwise specified, we choose $c$ equal to the mean equatorial radius $R_{e q}=(a+b) / 2$ and a rotation axis along $(O z)$, as in the experimental setup. In all simulations, the fluid is initially at rest and we suddenly impose at time $t=0$ a constant angular rate $\Omega$ such that the tangential velocity along the deformed boundaries in each plane perpendicular to the rotation axis, is equal to $\Omega\left(a^{\prime}+b^{\prime}\right) / 2$, where $a^{\prime}$ and $b^{\prime}$ are the axes of the elliptic boundary in this plane. In the following, results are non-dimensionalised using the mean equatorial radius $R_{e q}$ as the length scale and $\Omega^{-1}$ as the time scale. Then five dimensionless numbers are used to fully describe the system: the Ekman number $E=v /\left(\Omega R_{e q}^{2}\right)$, where $v$ is the kinematic viscosity of the fluid, the ellipticity $\varepsilon=\left(b^{2}-a^{2}\right) /\left(a^{2}+b^{2}\right)$ of the elliptical deformation, the aspect ratio $c / b$ which quantifies the oblateness of the ellipsoid, and finally the inclination $\theta$ and declination $\phi$ of the rotation axis. The problem numerically solved is then described by the following system of dimensionless equations:

$$
\frac{\partial \mathbf{u}}{\partial t}+\mathbf{u} \cdot \nabla \mathbf{u}=-\nabla p+E \Delta \mathbf{u}-2 \mathbf{\Omega}_{c}^{*} \times \mathbf{u}
$$

$$
\nabla \cdot \mathbf{u}=0
$$


where the no-slip boundary conditions are used for the fluid. Note that we work is the reference frame where the ellipsoidal shape is at rest, which is the inertial frame of reference in most of the paper. The Coriolis force $-2 \boldsymbol{\Omega}_{c}^{*} \times \boldsymbol{u}$ is only used in section 3.2 where the whole triaxial ellipsoid is submitted to a global rotation at $\Omega_{c}^{*} \boldsymbol{e}_{z}$.

Usually, numerical studies of planetary cores benefit from their spherical geometry to use fast and precise spectral methods. In our case however, there is no simple symmetry. Our computations are thus performed with a standard finite element method widely used in engineering studies, which allows to deal with complex geometries, such as our triaxial ellipsoid, and to simply impose the boundary conditions. Note that a very efficient finite element method was recently introduced by [9], but it is up to now restricted to spheroidal geometries $(a=b)$. Using a commercial software 1 , an unstructured mesh with tetrahedral elements was created. The mesh element type employed is the standard Lagrange element $P 1-P 2$, which is linear for the pressure field but quadratic for the velocity field. Note that no stabilization techniques have been used in this work. We use the so-called Implicit Differential-Algebraic solver (IDA solver), based on backward differencing formulas [18]. At each time step the system is solved with the sparse direct linear solver PARDISO2.

The elliptical instability induces a three-dimensional destabilization of the initial two-dimensional elliptical streamlines. To study its global properties, it thus seems natural to introduce the mean value of the vertical velocity $W=\frac{1}{V} \iiint_{V}|w| \mathrm{d} \tau$, with $w$ the dimensionless vertical velocity and $V$ the volume of the ellipsoid. The typical evolution of $\mathrm{W}$ as a function of time is shown in figure 2 (a) for $E=1 / 500$ and $\varepsilon=0.317$. At $t=0$, the fluid is at rest in the ellipsoid, and the no-slip condition at the boundary proceeds to set the fluid in rotation. The first peak in W, just after $t=0$, is due to the Ekman pumping which appears during the spin-up stage, which typically takes place over the Ekman time $t_{E}=E^{-1 / 2} \Omega^{-1}$, much faster than the viscous time scale $t_{v}=R_{e q}^{2} / v=E^{-1} \Omega^{-1}$ [3]. For instance, in the case shown on figure 2 (a), the dimensionless Ekman time gives $\Omega t_{E} \approx 22$, which agrees with the numerical results. After this initial stage, the fluid is essentially in solid body rotation. From this state, the exponential growth of the instability can be seen, before an overshoot and a stationary saturation. Having defined the growth rate $\sigma$ of the elliptical instability as the time constant of the exponential growth, a convergence study on this growth rate is given in the figure2 (b). The number of degrees of freedom (DoF) used in most of the simulations of this work ranges between $4 \cdot 10^{4} \mathrm{DoF}$ and $7 \cdot 10^{4} \mathrm{DoF}$, depending on the ellipticity and the Ekman number, in order to reach a compromise between a good convergence (see figure 2 (b)) and a reasonable CPU time.

\subsection{Validation of our numerical simulations}

A first visual validation is done on the shape of the flow in comparing the experimental visualisation on figure 3 (a) with the numerical simulation on figure 3(b). As can be seen, we recover the classical S-shape of the spin-over mode, which induces an additional solid body rotation of the fluid around the axis of maximum strain, i.e. perpendicular to the imposed rotation axis $(O z)$ and at an angle of about $45^{\circ}$ compared to the deformation axis $(O x)$ [27]. In order to quantitatively validate the numerical model, the evolution of the growth rate of the instability is compared in figure 4 to the linear theory given in [27] for small ellipticities :

$$
\frac{\sigma}{\varepsilon}=\frac{1}{2}-K \frac{\sqrt{E}}{\varepsilon},
$$

where $K$ is a constant equal to $K=2.62$ in the limit of small $\varepsilon([19,27])$. Note that the second term on the right hand side of the equation 3 corresponds to the viscous damping of the growth rate due to the presence of Ekman layers near boundaries [see [25, 19]. The expression (3) allows to define a critical Ekman number for the onset of the instability equal to

$$
E_{c}=\left(\frac{\varepsilon}{2 K}\right)^{2}
$$

Close to the threshold, the numerical results closely follow the linear analysis, for values of ellipticity as large as $\varepsilon \approx 0.5$. Note in particular that all curves for various $(\varepsilon, E)$ superimpose providing that $\sigma / \varepsilon$ is expressed as a function

\footnotetext{
${ }^{1}$ COMSOL Multiphysics ${ }^{\circledR}$

${ }^{2}$ www.pardiso-project.org
} 
of $\sqrt{E} / \varepsilon$. This is especially interesting in order to apply our results to planets, whose very small $E$ are not directly accessible by our numerical tool, but can be compensated for by large $\varepsilon$ in simulations.

Finally, for very large ellipticities $(\varepsilon>0.5)$, figure 4 shows that the growth rate decreases toward zero. The elliptical instability finally disappears for ellipticities greater than a critical value which depends on the Ekman number. The position of the maximum for the variation of $\sigma$ with the ellipticity is around $\varepsilon=0.5$, whatever the Ekman number is. This indicates that the decrease is probably due to geometrical effects related to the large value of $\varepsilon$, rather than to any viscous attenuation.

\section{Systematic numerical studies of geophysical complexities}

\subsection{Influence of the length of the ellipsoid along the rotation axis}

Having validated our numerical code, we can investigate the influence of the aspect ratio $c / b$ on the instability for a given ellipticity in the equatorial plane and a given rotation rate around the axis $(O z)$. This is directly related to geoand astro-physical flows as oblateness of planets, like the Earth for instance, is most of the time much stronger than its tidal deformation, meaning that the rotation axis is also the smallest one. The situation is even more pronounced in certain stars, as for instance Regulus A whose diameter is about 32\% greater at the equator than the distance between its poles [44].

Early theoretical work on this aspect was done by [21], who considered the inertial wave basis of an oblate spheroid $(a=b)$ and calculated the first 60 subharmonic exact resonances and their growth rate for small ellipticities depending on the value of $c$ [see also 23, for a special application to the case of Io]. An explicit theoretical answer for the growth rate has also been found by [15], starting from the base flow

$$
\mathbf{u}_{\mathbf{b}}=-\frac{a}{b} y \mathbf{e}_{\mathbf{x}}+\frac{b}{a} x \mathbf{e}_{\mathbf{y}}
$$

and looking for inviscid perturbations that are linear in space variables, corresponding to the classical spin-over mode. Here, $\mathbf{e}_{\mathbf{x}}$ and $\mathbf{e}_{\mathbf{y}}$ are respectively the unit vectors of $(O x)$ and $(O y)$. In an open domain, the base flow (5) corresponds to elliptical streamlines with an ellipticity $\varepsilon=\left(b^{2}-a^{2}\right) /\left(b^{2}+a^{2}\right)$ as in our numerical model, but with a variable tangential velocity. As shown in figure 5 this base flow is a very good approximation of our configuration where we impose a constant tangential velocity on the elliptical boundary, outside a small boundary layer close to the external wall where recirculation cells take place. For such a flow, the inviscid growth rate determined by [15] is:

$$
\sigma=\sqrt{\frac{\left(b^{2}-c^{2}\right)\left(c^{2}-a^{2}\right)}{\left(b^{2}+c^{2}\right)\left(a^{2}+c^{2}\right)}}
$$

Note that this theoretical growth rate is valid for $a \leq c \leq b$ only and is zero for $c=b$ or $c=a$. Then the maximal theoretical growth rate $\sigma_{\max }=\frac{b-a}{a+b}$ is obtained for $c=\sqrt{a b}$. One can notice that expression (3) leading to $\sigma=\frac{\varepsilon}{2}$ in the inviscid case is recovered with $a=R_{e q}+s$ and $b=R_{e q}-s$ in the limit $\varepsilon \rightarrow 0$. Note also that the experimental choice $c=(a+b) / 2$, equivalent to $c=\sqrt{a b}$ for small ellipticities, results in the growth rate remaining very close to the maximum; for instance, even for an ellipticity of 0.7 , the difference between the growth rate calculated for $c=\frac{a+b}{2}$ and the maximum value is only $2 \%$. The numerical results are presented in figure 6 In order to compare them with the inviscid analytical prediction (6), a viscous damping term $-K \sqrt{E}$ is added to the expression of the inviscid growth rate, similarly to the classical expression (3). Excellent agreement is then found for $a \leq c \leq b$, again validating our approach. However, a slightly different constant $K=2.5$ (instead of 2.62 in (6), valid in the limit of small $\varepsilon$ ) allows a better fit of our data. For strong deformations, this constant clearly depends on the considered ellipticity; for instance, a similar study performed at $\varepsilon=0.42$ implies $K=2.78$. Nevertheless, one can notice that $K$ always remains about the same order of magnitude. One can also notice that because of the scattering in the numerical results presented in figure 4 (a), such a constant $K=2.5$ works equally well. Hence in the following, we systematically use $K=2.5$.

In addition to the verification of the theoretical law of [15], we are now in the position of exploring the range outside $a \leq c \leq b$, where other modes may grow that are not necessarily linear in space variables. As shown in figure 6, different modes of the elliptical instability, characterized by their main frequency of oscillation, appear depending on the ratio $\mathrm{c} / \mathrm{b}$. In this view, the variation of the oblateness is similar to the variation of the aspect ratio in the case of an 
elliptically deformed cylinder [11]. Since the elliptical instability comes from the parametric resonance of two inertial waves of azimuthal wave number $m$ and $m+2$ with the underlying strain field, the corresponding mode is written $(m, m+2)$ and is related to a pulsation of frequency $\omega_{\text {mode }}=m+1$. For instance, the spin-over mode corresponds to the stationary mode $(-1,1)$ with half a wavelength along the axis of rotation. According to figure 6 (b), the mode $(1,3)$ can be observed when $c<b$ and the mode $(0,2)$ when $c>a$. For even larger aspect ratio, the mode $(-1,1)$ with a larger wavenumber than the spin-over takes place. Note however that because of the geometrical confinement, no stationary mode can be excited for $c<b$.

\subsection{Influence of a background rotation}

In geo- and astrophysics, the tidal deformation of a given body (planet, moon or star) is also in rotation because of the orbital motion of the companion body. The influence of this background rotation on the development of the elliptical instability has been studied theoretically, using short-wavelength analysis [8, 33, 35] or normal mode analysis [15, 21], and also numerically for specific vortices such as Stuart vortices [34, 50] or Taylor-Green vortices [57]. It has been studied experimentally in deformed cylinders [60, 32] and ellipsoids [4, 31]. All these works show that the background rotation has a stabilizing effect on cyclones and a destabilizing effect on anticyclones, except when the background rotation almost compensates for the flow rotation, in which case the elliptical instability disappears.

The theoretical expression of the growth rate for perturbations that are linear in space variables (i.e. corresponding to the classical spin-over mode) can be readily obtained by following the same method as [15], but taking into account an additional Coriolis force coming from the background rotation at a given angular velocity $\Omega_{c}=\Omega_{c}^{*} \Omega$ :

$$
\sigma=\sqrt{\frac{\left(b^{2}-c^{2}+2 \Omega_{c}^{*} a b\right)\left(c^{2}-a^{2}-2 \Omega_{c}^{*} a b\right)}{\left(b^{2}+c^{2}\right)\left(a^{2}+c^{2}\right)}} .
$$

Actually, this is a particular case of the more general stability analysis detailled in [7] for a precessing triaxial ellipsoid. The existence range of this mode is now between $c=b \sqrt{1+2 \Omega_{c}^{*} a / b}$ and $c=a \sqrt{1+2 \Omega_{c}^{*} b / a}$ and the maximal theoretical growth rate is now exactly obtained for $\frac{c^{2}}{a b}=\Omega_{c}^{*}+\sqrt{1+\Omega_{c}^{*}\left(a / b+b / a+\Omega_{c}^{*}\right)}$. In our numerical model, the addition of such a Coriolis force is straightforward and numerical results are presented in figure 7 (a). Again, they compare well with the theoretical prediction, providing that the viscous damping term, $-2.5 \sqrt{E}$, found in section 3.1 is added to the inviscid expression (7). Other modes are selected by the Coriolis force outside of the spin-over mode resonance band, as already observed experimentally by [31].

Finally, we can investigate how the oblateness and the background rotation interact with each others. Considering for instance an oblate ellipsoid with a ratio $c / b$ such as the mode $(1,3)$ is selected in absence of background rotation, the spin-over mode is recovered when decreasing $\Omega_{c}$, as shown in figure7(b). More generally, we find that the $(-1,1)$ mode is the most unstable one in the anticyclonic domain up to a value $\Omega_{c} / \Omega_{t o t} \lesssim-1$ where the flow restabilises, in agreement with the conclusions of [31].

\subsection{Influence of the obliquity of the ellipsoid}

In planetary cores, tidal deformations are aligned with the orbital plane rather than with the equatorial plane, which means that they are not orthogonal to the rotation axis. Previous works do not take into account this phenomenon, even though the obliquity can be significant, e.g. $23^{\circ} 26^{\prime}$ for the Earth. In this section, we thus investigate the effect of obliquity for the first time and consider that, as a first approximation, the shape of the body remains a triaxial ellipsoid.

In the numerical model, the rotation axis is tilted and oriented along the unit vector

$$
\mathbf{k}_{\mathbf{c}}=(\cos (\phi) \sin (\theta), \sin (\phi) \sin (\theta), \cos (\theta)),
$$

where $\phi$ is its azimuth angle and $\theta$ its colatitude angle in spherical coordinates (fig. 1). In assuming for example that the rotation axis is tilted in the $(O x z)$ plane (i.e. $\phi=0)$, we can study how the development of the elliptical instability changes depending on the obliquity $\theta$. For $\theta=0$, one recovers the usual configuration with $\varepsilon=\left(b^{2}-a^{2}\right) /\left(b^{2}+a^{2}\right)$ and an aspect ratio $c / b$. For $\theta=\pi / 2$, one recovers results from section 3.1 in exchanging $a$ and $c$, i.e. $\varepsilon=\left|b^{2}-c^{2}\right| /\left(b^{2}+c^{2}\right)$ and the aspect ratio is now $a / b$. In between, streamlines in planes perpendicular to the rotation axis are also elliptical, but their centers are not located along the rotation axis anymore, except in the equatorial plane $z=0$. Besides the effective 
ellipticity measured in each plane now depends on $z$, the maximum being reached in the equatorial plane $z=0$. Seen from this equatorial plane, the apparent length of the ellipsoid along the rotation axis is $\tilde{c}=\left(\frac{\sin ^{2} \theta}{a^{2}}+\frac{\cos ^{2} \theta}{c^{2}}\right)^{-\frac{1}{2}}$, whereas the great and small axes of the elliptical streamlines are respectively $\tilde{b}=b$ and $\tilde{a}=\left(\frac{\cos ^{2} \theta}{a^{2}}+\frac{\sin ^{2} \theta}{c^{2}}\right)^{-\frac{1}{2}}$. One can then estimate the growth rate of the instability using formulas (3) or (6) with these apparent lengths. Numerical results for the spin-over mode compared with these approximations are presented in figure 8 Results agree well for obliquity up to $\theta \sim 20^{\circ}$. Then, the numerical growth rate significantly differs, probably because the geometry is very far from an apparent ellipsoid in rotation around one of its principal axes. It remains however between the two expressions proposed. Note that for the Ekman number studied here, we do not see the tidal instability reappearing around $\theta \sim 90^{\circ}$, which will however be the case at smaller Ekman number: as seen in section 3.1, in this case other modes could appear because of the modified oblateness.

\section{Scaling laws for global quantities of geophysical interest}

\subsection{Scaling law for the amplitude of the flow driven by the instability}

The amplitude of the flow driven by the instability at saturation remains up to now an open question, mainly because it is determined by strong non-linear interactions. It is however an important quantity for geo- and astrophysical applications, since it allows us to evaluate the influence of elliptical aspects compared to the other relevant ingredients of planetary dynamics, such as convection. In order to study the amplitude of the flow driven by the instability at saturation, we define the amplitude $A^{*}$ by the maximum value over the volume $V$ :

$$
A^{*}=\max _{V}\left\|\mathbf{u}-\mathbf{u}_{\mathbf{b}}\right\|
$$

where $\mathbf{u}$ is the dimensionless velocity field and $\mathbf{u}_{\mathbf{b}}$ is the theoretical base flow defined in section 3.1 The evolution of $A^{*}$ as a function of the Ekman number is shown in figure 9 (a) for various ellipticities: it can be seen that $A^{*}$ is not zero below the threshold of the elliptical instability because of the differences around the outer bound between the theoretical base flow and the flow numerically obtained (see figure 5). Actually, the difference is maximal on the boundary, along the smallest equatorial axis where the theoretical velocity is maximal. There, the corresponding amplitude $\lambda_{(\varepsilon)}$ can be calculated theoretically :

$$
\lambda_{(\varepsilon)}=\frac{b}{\frac{a+b}{2}}-1=\frac{2}{1+\sqrt{\frac{1-\varepsilon}{1+\varepsilon}}}-1
$$

which only depends on $\varepsilon$. One then defines the amplitude of the flow driven by the instability by $A=A^{*}-\lambda_{(\varepsilon)}$, which is equal to 0 below the threshold (fig. 9 (b)).

Far from threshold, a secondary instability appears, which induces a secondary dynamics superimposed on the primary state : e.g. typically, for $\varepsilon=0.317, E=1 / 1500, c=(a+b) / 2$, the spinover mode is no more stationary, and the flow is slightly oscillating around the spinover mean flow, at a pulsation $\omega_{\text {sec }} \approx 1.4$. Note that this is in agreement with [22] which predicts that the primary elliptical instability should saturate and be stable only in a small strain window $\varepsilon-\varepsilon_{c}=O(E)$. Thus, the amplitude $A$ has to be averaged in time to smooth out the small scale fluctuations. Since those small-scale fluctuations take place on a typical time scale comparable to one revolution $2 \pi / \Omega$, whereas the characteristic time for the tidal instability is the inverse of the growth rate, i.e. $(\Omega \varepsilon / 2)^{-1}$, the averaging is performed about a typical time $\Omega^{-1} \sqrt{4 \pi / \varepsilon}$ corresponding to the geometrical mean of these two extreme values.

With this definition, figure 9 (b) shows that all results collapse on the same generic law above the threshold providing that we use the variable $E_{c} / E-1$. Far from threshold, the amplitude seems to saturate around 1 , which means that the velocities generated by the tidal instability are comparable to the imposed boundary rotation.

One can notice that near the threshold, a square root $A \approx 0.6 \sqrt{E_{c} / E-1}$ fits the results, which is in agreement with a pitchfork bifurcation. Actually, this scaling law can be obtained analytically, starting from the simple model used in [27] to describe the viscous non-linear evolution of the spinover mode. This model, first introduced by [20] and [49] for an inviscid solid-body rotation in a spheroid, reduces to:

$$
\dot{\omega}_{x}=-\alpha\left(1+\omega_{z}\right) \omega_{y}-v_{s o} \omega_{x}
$$




$$
\begin{gathered}
\dot{\omega}_{y}=-\beta\left(1+\omega_{z}\right) \omega_{x}-v_{s o} \omega_{y} \\
\dot{\omega}_{z}=\varepsilon \omega_{x} \omega_{y}-v_{e c} \omega_{z}+v_{n l}\left(\omega_{x}^{2}+\omega_{y}^{2}\right)
\end{gathered}
$$

where $\omega$ is the rotation vector of the spinover mode, $\alpha=\frac{\varepsilon}{2-\varepsilon}$ and $\beta=\frac{\varepsilon}{2+\varepsilon}$. The damping terms are given by theory (no adjustable parameter): $v_{s o}=2.62 \sqrt{E}$ is the linear viscous damping rate of the spinover mode (first calculated by [12]), $v_{e c}=2.85 \sqrt{E}$ is the linear viscous damping of axial rotation and $v_{n l}=1.42 \sqrt{E}$ is the viscous boundary layer effect on the non-linear interaction of the spinover mode with itself (see [12]). Even if this model does not take into account all the viscous terms of $O(\sqrt{E})$ or the non-linear corrections induced by the internal shear layers, it satisfyingly agrees with experiments, regarding the growth rate as well as the non-linear saturation [27]. Thus, this model can be used with confidence to describe the viscous non-linear evolution of the spinover mode.

After little algebra, we obtain a non-trivial stationary state for $\varepsilon>2 v_{s o}$, given by:

$$
\begin{gathered}
\omega_{x}= \pm \sqrt{\frac{v_{e c}\left[\sqrt{\alpha \beta}-v_{s o}\right]}{\beta \varepsilon-v_{n l}\left[\sqrt{\alpha \beta}+\beta^{2} / \sqrt{\alpha \beta}\right]}} \approx \pm \sqrt{\frac{v_{e c}\left[\varepsilon-2 v_{s o}\right]}{\varepsilon^{2}-2 v_{n l} \varepsilon}} \\
\omega_{y}=\mp \sqrt{\frac{v_{e c}\left[\sqrt{\alpha \beta}-v_{s o}\right]}{\alpha \varepsilon-v_{n l}\left[\sqrt{\alpha \beta}+\alpha^{2} / \sqrt{\alpha \beta}\right]}} \approx \mp \sqrt{\frac{v_{e c}\left[\varepsilon-2 v_{s o}\right]}{\varepsilon^{2}-2 v_{n l} \varepsilon}} \approx \mp \omega_{x} \\
\omega_{z}=\frac{v_{s o}}{\varepsilon} \sqrt{4-\varepsilon^{2}}-1 \approx \frac{2 v_{s o}}{\varepsilon}-1
\end{gathered}
$$

where approximations are done assuming $\varepsilon \ll 1$. Now, the amplitude $A$ corresponds to the norm of the flow $\boldsymbol{\omega} \times \boldsymbol{r}$ driven by the spinover mode. Then, near the threshold $\left(\varepsilon \approx 2 \cdot 2.62 \sqrt{E_{c}}\right.$ and $\left.E_{c} / E-1 \ll 1\right)$, we obtain:

$$
A \approx \sqrt{\frac{v_{e c}}{2\left(v_{s o}-v_{n l}\right)}\left(\frac{E_{c}}{E}-1\right)} \approx 1.1 \sqrt{\frac{E_{c}}{E}-1}
$$

which is in good agreement with the numerical fit.

\subsection{Scaling law for the viscous dissipation by the instability}

As explained for instance in [51] or in [31], the energy dissipated by tides is a primordial quantity which directly influences the orbital evolution and rotational history of a binary system during its synchronization. It is however poorly known [e.g. 63, 59]. In most traditional models, fluid dissipation in the planetary core is supposed to be negligible. However this may not be the case when the elliptical instability is excited at a rather large amplitude, inducing important shears between the bulk of the fluid and the boundary. Our purpose here is to systematically quantify the variation of this dissipation with the ellipticity and the Ekman number.

The dissipated power balance of the incompressible flow in the ellipsoid is given by:

$$
\iiint_{V} \frac{\partial}{\partial t}\left(\rho \frac{\tilde{u}^{2}}{2}\right) \mathrm{d} \tau=\iint_{S}\left(\overline{\overline{\boldsymbol{\sigma}}}_{v} \cdot \mathbf{n}\right) \cdot \tilde{\mathbf{u}} \mathrm{d} s-\iiint_{V} \overline{\overline{\boldsymbol{\sigma}}}_{v}: \nabla \tilde{\mathbf{u}} \mathrm{d} \tau
$$

where $\overline{\bar{\sigma}}_{v}=\eta\left(\nabla \tilde{\mathbf{u}}+{ }^{t} \nabla \tilde{\mathbf{u}}\right)$ is the viscous stress tensor of the newtonian fluid, $S$ the surface of the ellipsoid, $\tilde{\mathbf{u}}=\Omega R_{e q} \mathbf{u}$ is the dimensionalized velocity field, $\rho$ the volumic mass and $\eta$ the dynamic viscosity of the fluid. In this section, we focus on the stationary spinover mode and then the two terms on the right side of equation (17) balance each others: the first one allows to maintain the rotation of the fluid by the no-slip conditions on the boundary while the second one is the volume dissipation $P$ of the fluid. In the following, we use as a power scale the dissipated power during the spin-down stage of the equivalent sphere of radius $R_{e q}$ and moment of inertia $I_{\Delta}\left(I_{\Delta}=2 / 5 M R^{2}\right.$ for an homogeneous sphere of mass $M$ and radius $R$ ), i.e. the kinetic energy of rotation $E_{c}=\frac{1}{2} I_{\Delta} \Omega^{2}$ divided by the Ekman time $t_{E}$. Note 
that most of the dissipated power comes naturally from the boundary layers: its determination is thus a challenging task from a numerical point of view, and extra care must be taken regarding the convergence of the simulations, as shown for instance in figure 2 (b).

Below the threshold, the dissipated power is not zero because of the recirculation patterns of our base flow (see figure 5). This dissipation is due to the flow driven by the ellipticity, which scales as $\varepsilon \Omega R$ for small ellipticities. Then, the dimensionalized dissipated power below the threshold simply scales as

$$
\iiint_{V} \overline{\overline{\boldsymbol{\sigma}}}_{v}: \nabla \tilde{\mathbf{u}} \mathrm{d} \tau \sim \eta \varepsilon^{2} \Omega^{2} R^{3}
$$

which is confirmed by the dissipated power measured in our numerical simulations below the threshold.

Far from threshold, the model proposed by [31] considers that the spin-over mode simply corresponds to a supplementary solid body rotation $\boldsymbol{\Omega}_{\text {SO }}$ outside the outer viscous boundary layer of thickness $h=\xi \sqrt{v / \Omega_{S O}}$, $\xi$ being a constant of order 1 . Then, the dissipation is only located in this boundary layer, where the fluid rotation has to match the imposed velocity conditions at the outer boundary. With this simple model, the torque of the container on the fluid is $\mathbf{C}^{\mathbf{m} / \mathbf{c}}=-2 M v \frac{R}{h} \boldsymbol{\Omega}_{\mathbf{S O}}$, and the power dissipated by the system is $P=-2 M v \frac{R}{h} \Omega_{S O}{ }^{2}$, which can also be written $P=-\frac{2}{\xi} M R \sqrt{v} \Omega^{5 / 2} A^{5 / 2}$, where $A$ is the dimensionless spin-over mode amplitude studied in the previous section. The dimensionless power given by this model can finally be stated as

$$
P_{\text {dissip }}=\frac{|P|}{\frac{1}{2} I_{\Delta} \Omega^{3} \sqrt{E}}=\frac{10}{\xi} A^{5 / 2},
$$

where, according to the previous section, $A$ is about 1 in the small Ekman number limit studied here.

The evolution of $P_{\text {dissip }}$ measured in our numerical model is shown in figure 10. It confirms the behavior predicted by (19) with a saturation far from threshold and $\xi \approx 1$. This behavior is especially interesting for planetary applications, as will be studied in the following section.

\section{Orders of magnitude for planetary applications}

From a geophysical point of view, the results from the previous sections can be used to derive the orders of magnitude involved in planetary cores. Regarding the jovian moon Io, which is clearly unstable to the tidal instability and where the strong tidal dissipation is a topical question [30], our numerical study confirms the first trends given by [31], with however a numerically determined scaling factor of the order $1 / \xi \approx 1$ in the power dissipation estimates. Moreover, the most important result comes from the oblateness effects. Indeed, according to the data given in [23], $c / b \sim 0.995$ and $a / b>0.999$, which means that the excited mode of the tidal instability in Io cannot be the spinover mode, but has to be oscillatory. Note that this result is not modified when the background rotation is taken into account.

A similar analysis can be done for the early Earth, with a Moon two times closer than today. In this case, the length of the day was around 10 hours according to [59] and the lunar tides were around eight times stronger, whereas the solar tides were about the same. Then, the actual tidal amplitude of $50 \mathrm{~cm}$ allows to estimate the ellipticity of the early Earth: $\varepsilon \sim 10^{-6}$. In considering a similar but totally molten core, we estimate $E_{c} / E-1 \sim 70$, which means that the early Earth's core was clearly unstable to tidal instability, and that the tidal instability was then at saturation. Moreover, with an orbital period for the Moon $(1 / 2)^{3 / 2}$ shorter than the actual moon (Kepler's Third Law), the dissipated power given by the model was around $P \sim 10^{18} \mathrm{~W}$. This estimation seems huge in comparison with the present dissipation by tidal friction $\left(\sim 3.75 \cdot 10^{12} \mathrm{~W}\right.$ according to [46]) but actually, it simply suggests that the Earth-Moon system was then in rapid evolution. Once again, the possible mode of the tidal instability was not the spin-over mode neither any stationary $(-1,1)$ mode, considering that the oblateness of the rapidly rotating early Earth was larger than the actual oblateness. In fact, we expect stationary modes to be only marginally excited in geophysical systems, limited to very peculiar configuration regarding the oblateness and the rotation of the tidal bulge.

Note that computations similar to the ones presented here for an ellipsoidal geometry have been performed for an ellipsoidal shell in order to study the influence of a solid inner planetary core. Similar conclusions are then found regarding the physics of the tidal instability. Only a slightly larger dissipation term due to the presence of an inner viscous boundary layer has to be taken into account. 


\section{Conclusion}

This paper presents the first systematic numerical study of the tidal instability in a rotating ellipsoid. The numerical approach is a powerful tool to complete the knowledge derived from previous theoretical and experimental studies. The effects of oblateness, background rotation and obliquity, which lead to the selection of various instability modes, have been studied. We have also defined scaling laws regarding the amplitude of the flow driven by the instability and its viscous dissipation, which confirm the primordial role played by tidal effects at a planetary scale.

Another fundamental geophysical issue regarding the elliptical instability is whether or not it can drive a planetary dynamo. The results presented herein show that the numerical approach is able to faithfully capture the physics of the hydrodynamic elliptical instability. Thus, the present work provides a solid basis to study the full MHD problem encountered in planetary core flows undergoing tidal instability. These flows can only be studied numerically due to their inherent complexity. Therefore, a next step will be to introduce the effects of a magnetic field in our model.

\section{References}

[1] Aldridge, K., Seyed-Mahmoud, B., Henderson, G., van Wijngaarden, W., 1997. Elliptical instability of the Earth's fluid core. Phys. Earth Planet. Int. 103, 365-74.

[2] Bayly, B. J., 1986. Three-dimensional instability of elliptical flow. Phys. Rev. Lett. 57, 2160 - 2163

[3] Benton, E.R., Clark, A., 1974. Spin-Up. Annu. Rev. Fluid. Mech. 6, 257-280.

[4] Boubnov, B. M., 1978. Effect of Coriolis force field on the motion of a fluid inside an ellipsoidal cavity. Izv. Atmos. Ocean. Phys, $14,501-504$.

[5] Cadot, O., Douady, S., Couder, Y., 1995. Characterization of the low pressure filaments in three-dimensional turbulent shear flow. Phys. Fluids 7, 630-646.

[6] Cébron, D., Maubert, P., Le Bars, M., 2010. Tidal instability in a rotating and differentially heated ellipsoidal shell. Geophys. J. Int. In revision.

[7] Cébron, D., Le Bars, M., Meunier, P., 2010. Tilt-over mode in a precessing triaxial ellipsoid. Phys. Fluids. In revision.

[8] Craik, A. D. D., 1989. The stability of unbounded two- and three-dimensional flows subject to body forces: some exact solutions. J. Fluid Mech. 198, 275-292.

[9] Chan, K. H, Zhang, K., Liao, X., 2010. An EBE finite element method for simulating nonlinear flows in rotating spheroidal cavities. International Journal for Numerical Methods in Fluids Volume 63 Issue 3, Pages 395 - 414

[10] Eloy, E., Le Gal, P., Le Dizès, S., 2000. Experimental study of the multipolar vortex instability. Phys. Rev. Lett. 85, $145-166$.

[11] Eloy, E., Le Gal, P., Le Dizès, S., 2003. Elliptic and triangular instabilities in rotating cylinders. J. Fluid Mech. 476, 357-388.

[12] Greenspan, H. P., 1968. The Theory of Rotating Fluids, Cambridge University Press, Cambridge.

[13] Herreman, W., Le Bars, M., Le Gal, P., 2009. On the effects of an imposed magnetic field on the elliptical instability in rotating spheroids. Phys. Fluids 21, 046602.

[14] Gledzer, E. B., Ponomarev, V. M., 1977. Finite dimensional approximation of the motions of incompressible fluid in an ellipsoidal cavity. Izv. Atmos. Ocean. Phys. 13, 565-569.

[15] Gledzer, E. B., Ponomarev, V. M., 1992. Instability of bounded flows with elliptical streamlines. J. Fluid Mech. 240, 1-30.

[16] Gledzer, E. B., Dolzhansky, F. V., Obukhov, A. M., Ponomarev, V. M., 1975. An experimental and theoretical study of the stability of motion of a liquid in an elliptical cylinder. Izv. Atmos. Ocean. Phys. 11, 617-622.

[17] Haj-Hariri, H., Homsy, G. M., 1997. Three-dimensional instability of viscoelastic elliptic vortices. J. Fluid Mech. 353, 357-381.

[18] Hindmarsh, A. C., Brown, P. N., Grant, K. E., Lee, S. L., Serban, R., Shumaker, D. E., Woodward, C. S., 2005. SUNDIALS: Suite of Nonlinear and Differential/Algebraic Equation Solvers. ACM T. Math. Software, vol. 31, p. 363.

[19] Hollerbach, R., Kerswell, R. R., 1995. Oscillatory internal shear layers in rotating and precessing flows. J. Fluid Mech. 298, 327-339.

[20] Hough, S. S., 1895. The oscillations of a rotating ellipsoidal shell containing fluid. Phil. Trans. A 186, $469-506$.

[21] Kerswell, R. R., 1994. Tidal excitation of hydromagnetic waves and their damping in the Earth. J. Fluid Mech. 274, 219-241.

[22] Kerswell, R. R., 2002. Elliptical instability. Annu. Rev. Fluid. Mech. 34, 83-113.

[23] Kerswell, R. R., Malkus, W. V. R., 1998. Tidal instability as the source for Io's magnetic signature. Geophys. Res. Lett. 25, 603-6.

[24] Kelvin, L., 1880. Vibrations of a columnar vortex. Phil. Mag. 10, 155-168.

[25] Kudlick, M., 1966. On the transient motions in a contained rotating fluid. PhD thesis, MIT.

[26] Lacaze, L., Herreman, W., Le Bars, M., Le Dizès, S., Le Gal, P., 2006. Magnetic field induced by elliptical instability in a rotating spheroid. Geophys. Astrophys. Fluid Dyn. 100, 299-317.

[27] Lacaze, L., Le Gal, P., Le Dizès, S., 2004. Elliptical instability in a rotating spheroid. J. Fluid Mech. 505, 1-22.

[28] Lacaze, L., Le Gal, P., Le Dizès, S., 2005. Elliptical instability of the flow in a rotating shell . Phys. Earth. Planet. Int., 151, pp. 194-205

[29] Lacaze, L., Ryan, K., Le Dizès, S., 2007. Elliptic instability in a strained Batchelor vortex. J. Fluid Mech. 577, 341

[30] Lainey, V., Arlot, J. E., Karatekin, O., Van Hoolst, T., 2009. Strong tidal dissipation in Io and Jupiter from astrometric observations. Nature 459, 957-959.

[31] Le Bars, M., Lacaze, M., Le Dizès, S., Le Gal, P., Rieutord, M., 2010. Tidal instability in stellar and planetary binary system. Phys. Earth. Planet. Int., 178, Issues 1-2, January 2010, Pages 48-55.

[32] Le Bars, M., Le Dizès, S., Le Gal, P., 2007. Coriolis effects on the elliptical instability in cylindrical and spherical rotating containers. J. Fluid Mech. 585, 323-342.

[33] Leblanc, S., Cambon, C., 1997. On the three-dimensional instabilities of plane flows subjected to Coriolis force. Phys. Fluids 9 , 1307.

[34] Leblanc, S., Cambon, C., 1998. Effects of the Coriolis force on the stability of Stuart vortices. J. Fluid Mech. 356, 353-379. 
[35] Le Dizès, S., 2000. Three-dimensional instability of a multipolar vortex in a rotating flow. Phys. Fluids 12, $2762-74$.

[36] Le Dizès, S., Laporte, F., 2002. Theoretical predictions for the elliptic instability in a two-vortex flow. J. Fluid Mech. 471, 169-201.

[37] Leweke, T., Williamson, C. H. K., 1998. Three-dimensional instabilities in wake transition. Eur. J. Mech. B/Fluids 17, 571-586.

[38] Leweke, T., Williamson, C. H. K., 1998. Cooperative elliptic instability of a vortex pair. J. Fluid Mech. 360, 85-119.

[39] Lundgren, T. S., Mansour, N. N., 1995. Transition to turbulence in an elliptic vortex. J. Fluid Mech. 307, 43-62.

[40] Malkus, W. V. R., 1968. Precession of the Earth as the cause of geomagnetism. Science 160, 259-264.

[41] Malkus, W. V. R., 1989. An experimental study of global instabilities due to tidal (elliptical) distortion of a rotating elastic cylinder. Geophys. Astrophys. Fluid Dyn. 48, 123-134.

[42] Mason, D. M., Kerswell, R. R., 1999. Nonlinear evolution of the elliptical instability: an example of inertial wave breakdown. J. Fluid Mech. 396, 73-108.

[43] Meunier, P., Ehrenstein, U., Leweke, T., Rossi, M., 2002. A merging criterion for two-dimensional co-rotating vortices. Phys. Fluids 14, 2757-2766

[44] McAlister, H. A., Ten Brummelaar, T. A., Gies, D. R., Huang, W. , Bagnuolo, W. G., Shure, M. A., Sturmann, J., Sturmann, L., Turner, N. H., Taylor, S. F., Berger, D. H., Baines, E. K., Grundstrom, E., Ogden, C., Ridgway, S. T., van Belle, G., 2005. First results from the CHARA array. I. An interferometric and spectroscopic study of the fast rotator $\alpha$ Leonis (Regulus). The Astrophysical journal, vol. 628 (1), no1, pp. 439-452.

[45] Moore, D. W., Saffman, P. G., 1975. The instability of a straight vortex filament in a strain field. Proc. R. Soc. Lond. A 346, $413-425$.

[46] Munk, W., 1998. Abyssal recipes II: energetics of tidal and wind mixing. Deep Sea Res. Part I. Oceanographic Research, vol. 45, no12, pp. 1977-2010.

[47] Owen, J. M., Rogers, R. H., 1989. Flow and heat transfer in rotating disc systems (Vol. 1, Rotor-stator systems). Research Studies Press, Taunton, UK and John Wiley, NY.

[48] Pierrehumbert, R. T., 1986. Universal short-wave instability of two-dimensional eddies in an inviscid fluid. Phys. Rev. Lett. 57,2157 - 2159.

[49] Poincaré, R., 1910. Sur la précession des corps déformables. Bull. Astr. 27 (1910) 321.

[50] Potylitsin, S., Peltier, W. R., 1999. Three-dimensional destabilization of Stuart vortices: the influence of rotation and ellipticity. J. Fluid Mech. 387,205-226.

[51] Rieutord, M., 2003. Evolution of rotation in binaries: physical processes. Stellar Rotation, Proc. IAU Symp. $215,394-403$.

[52] Roy, M., Schaeffer, N., Le Dizès, S., Thompson M. C., 2007. Stability of a pair of co-rotating vortices with axial flow. Phys. Fluids 20, 094101.

[53] Seyed-Mahmoud, B., Aldridge, K. D., Henderson, G., 2004. Elliptical instability in rotating spherical fluid shells: application to Earth's fluid core. Phys. Earth Planet. Int. 142, 257-282.

[54] Seyed-Mahmoud, B., Henderson, G., Aldridge, K. D., 2000. A numerical model for elliptical instability of the Earth's fluid outer core. Phys. Earth Planet. Int. 117, 51-61.

[55] Shangli, O., Henderson, J. E., Motl, P. M., 2007. Further evidence for an elliptical instability in rotating fluid bars and ellipsoidal stars.Astrophys. J. 665,1074

[56] Sipp, D., Jacquin, L., 1998. Elliptic instability in two-dimensional flattened Taylor-Green vortices. Phys. Fluids $10,839$.

[57] Sipp, D., Lauga, E., Jacquin, L., 1999. Vortices in rotating systems: Centrifugal, elliptic and hyperbolic type instabilities. Phys. Fluids, vol. 11 , Issue $12,3716$.

[58] Thess, A., Zikanov, O., 2007. Transition from two-dimensional to three-dimensional magnetohydrodynamic turbulence. J. Fluid Mech., 579, p. $383-412$.

[59] Touma, J., Wisdom, J., 1994. Evolution of the Earth-Moon system. The Astr. J., vol. 108, no. 5, p. $1943-1961$.

[60] Vladimirov, V. A., Tarasov, V. F., Rybak, L. Ia., 1983. The stability of the elliptically deformed rotation of an ideal incompressible fluid in a Coriolis force field. Izv. Atmos. Ocean. Phys, 14, 501-504.

[61] Waleffe, F. A., 1990. On the three-dimensional instability of strained vortices. Phys. Fluids 2, 76-80.

[62] Widnall, S. E., Bliss, D., Tsai, C.-Y, 1974. The instability of short waves on a vortex ring. J. Fluid Mech. 66, 35-47.

[63] Williams, G. E., 2000. Geological constraints on the Precambrian history of Earth's rotation and the Moon's orbit. Reviews of Geophysics, Volume 38, Issue 1, p. 37-60. 


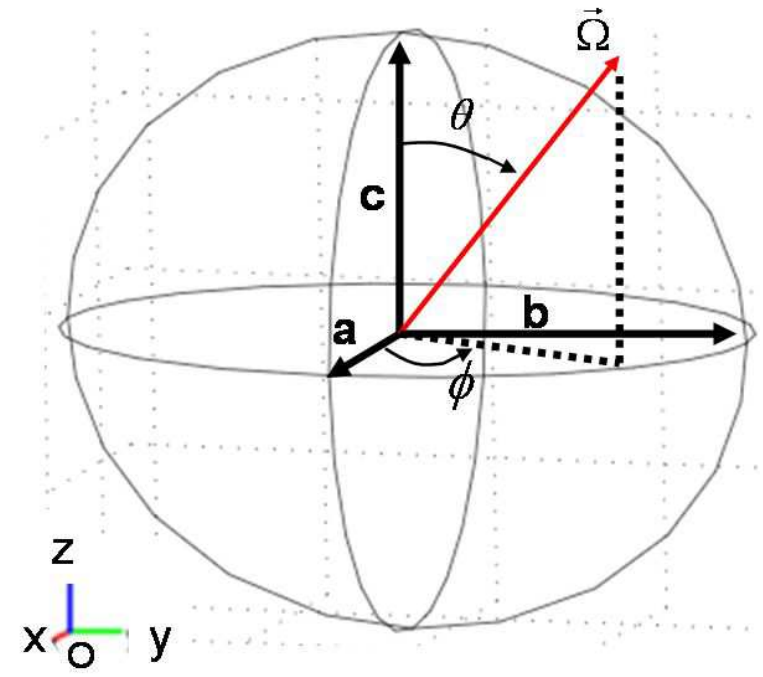

Figure 1: Sketch of the problem under consideration : a rotating flow inside a triaxial ellipsoid of axes $(a, b, c)$ related to a frame $(O x, O y, O z)$ with a constant tangential velocity all along the boundary in each plane perpendicular to the rotation vector $\mathbf{\Omega}$. 


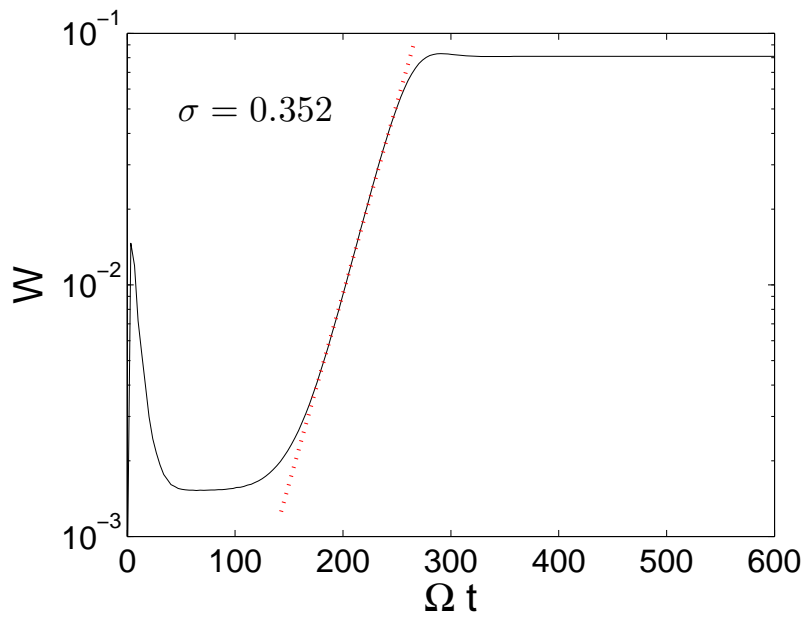

(a)

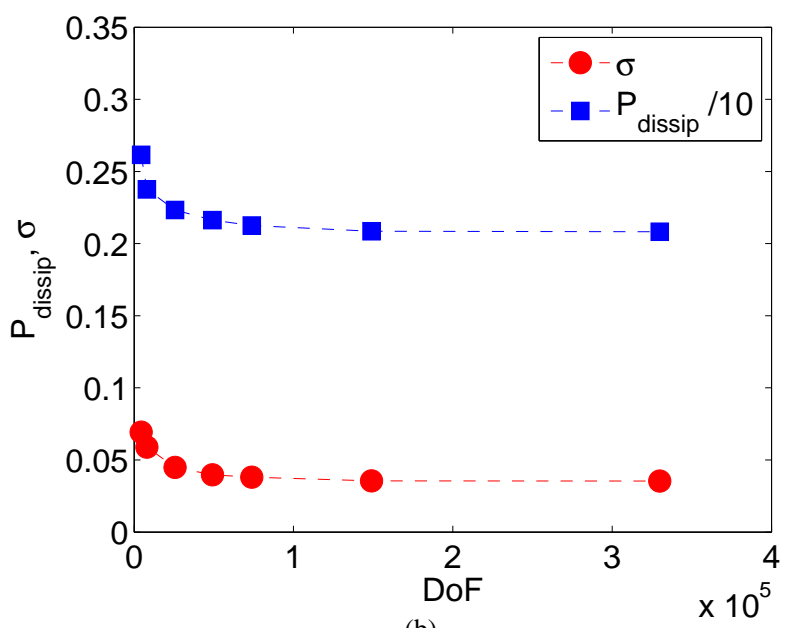

(b)

Figure 2: Simulations performed at $E=1 / 500$ and $\varepsilon=0.317$ for $c=\frac{a+b}{2}$. (a) Time evolution of the mean value of the vertical velocity, showing the spin-up phase $\left(\Omega t_{E} \approx 22\right.$ ), the exponential growth of the tidal instability (see also in dotted line the exponential fit with a growth rate $\sigma=0.352$ ) and its saturation. (b) Convergence with the number of degrees of freedom $(D o F)$ of the growth rate $\sigma$ and of the dissipated power $P_{\text {dissip }}$ at saturation. Results presented in (a) are computed for $42459 \mathrm{DoF}$. 


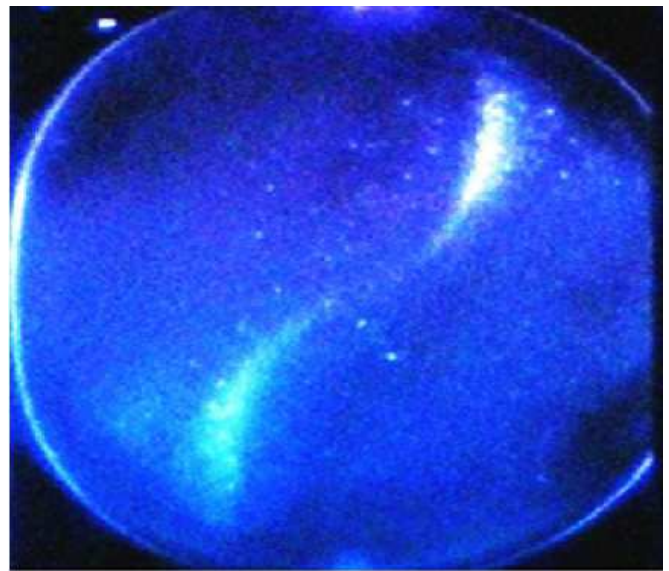

(a)

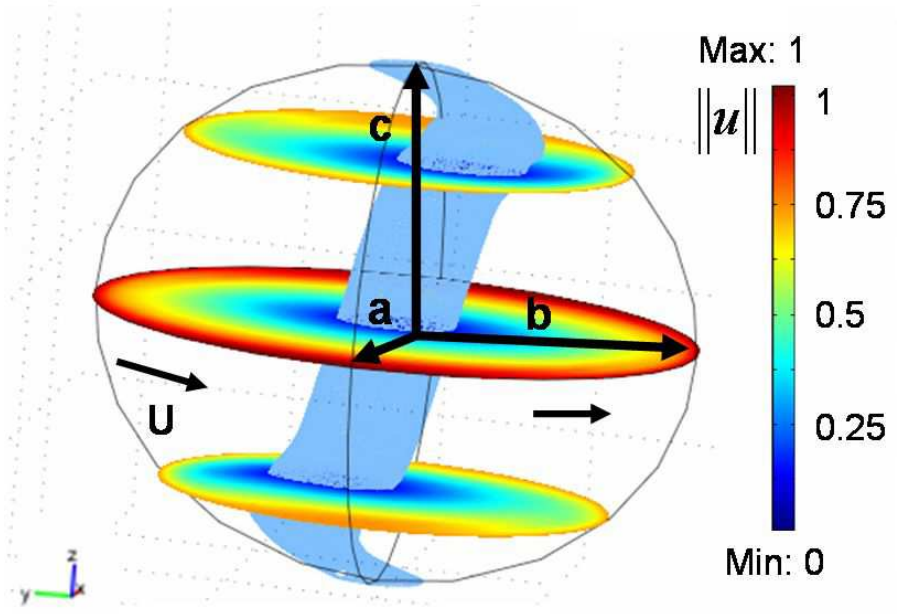

(b)

Figure 3: Validation of the numerical simulations. (a) Kalliroscopic visualization of the spin-over mode in the meridional plane of maximum shear for $E=1 / 4000$ and $\varepsilon=0.16$. The typical $S$ shape of the rotation axis is due to the combination of the main rotation imposed by the boundary and the spin-over mode. (b) Slices of the velocity field $\|\mathbf{u}\|$ and surface of iso-value $\|\mathbf{u}\|=0.15$ at saturation of the tidal instability for $E=1 / 344$, $\varepsilon=0.317, c=\frac{a+b}{2}$, and $49900 \mathrm{DoF}$. The classical $S$-shape of the spin-over mode is recovered. 


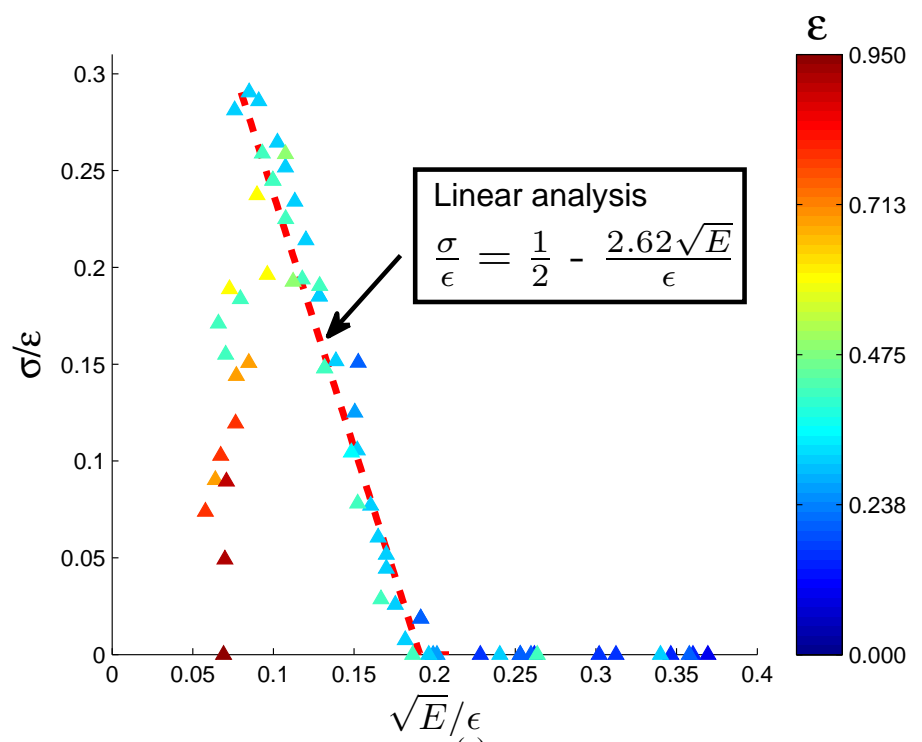

(a)

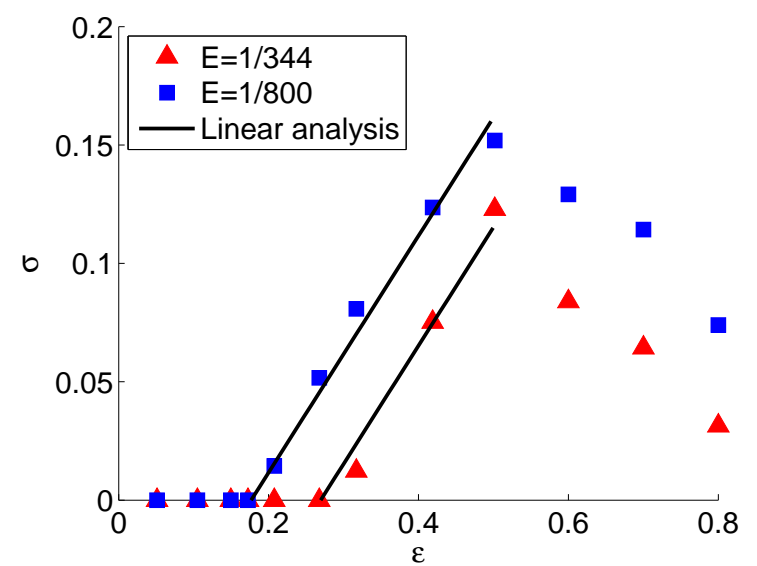

(b)

Figure 4: Validation of the numerical simulations. (a) Evolution of the growth rate for $\left(E \geq 1 / 2000, \varepsilon \leq 0.95\right.$, $\left.c=\frac{a+b}{2}\right)$ and comparison with the linear theory indicated by a dashed line. The coefficient 2.62 comes from [27] and is valid in the limit of small ellipticity. Good agreement is found for values of $\varepsilon$ up to 0.5 . (b) Evolution of the growth rate depending on the ellipticity for two values of the Ekman number $E=1 / 344$ and $E=1 / 800\left(\varepsilon \leq 0.8, c=\frac{a+b}{2}\right)$. As also seen in $(a)$, the growth rate agrees with the linear analytical analysis close to the threshold and then decreases for large values of the ellipticity. 


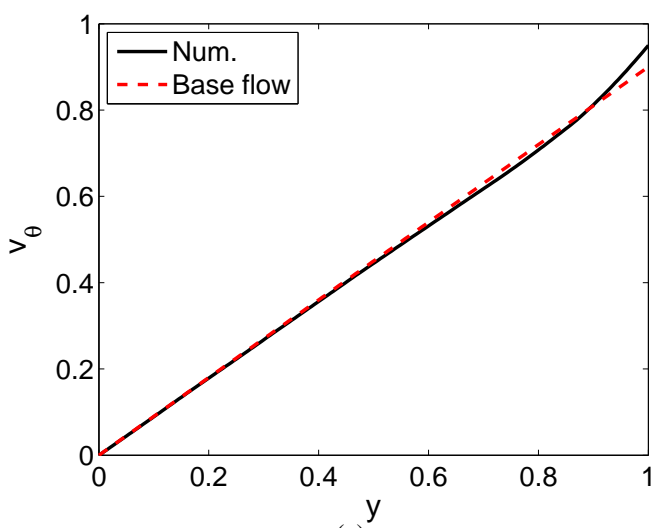

(a)

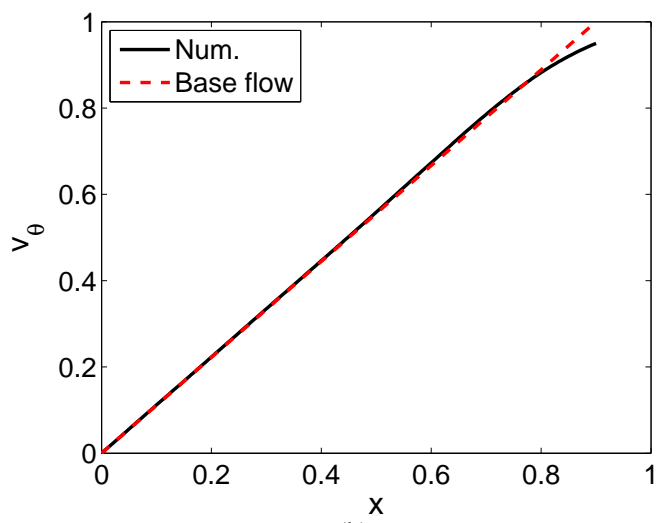

(b)

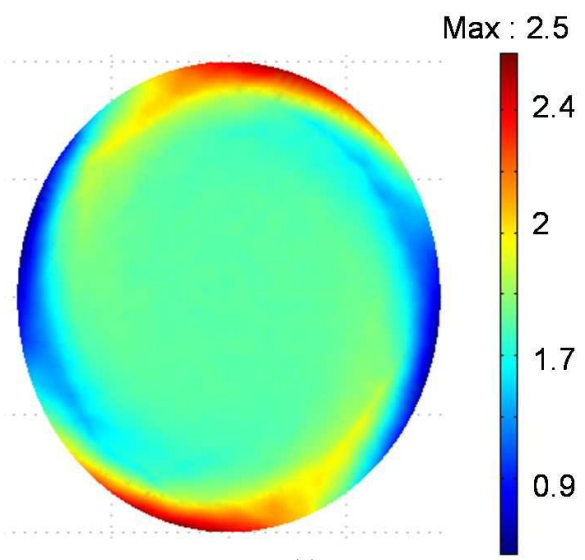

(c)

Figure 5: Azimuthal velocity $v_{\theta}$ for $E=1 / 95, \varepsilon=0.1, c=\frac{a+b}{2}$. (a) Slice along (Oy) at $x=0$. (b) Slice along (Ox) at $y=0$. (c) Slice in the equatorial plane of the vertical component of the vorticity. Dashed lines in (a) and (b) correspond to the theoretical base flow (5), which presents a variable tangential velocity along elliptical streamlines. Good agreement is found with the numerical results, except in the small outer viscous boundary layer, where recirculation cells take place to match the imposed constant velocity along the boundary. Note in particular that the maximum velocity is not reached at the boundary but within this viscous boundary layer. 


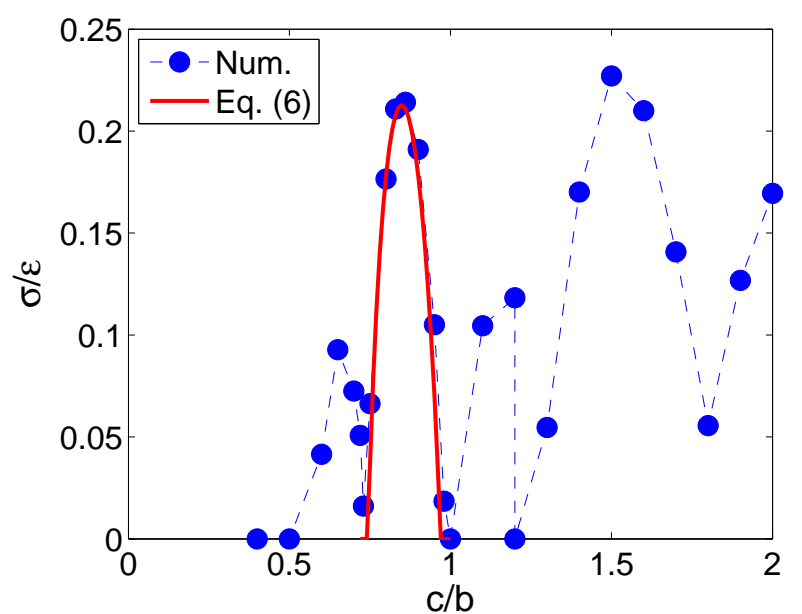

(a)

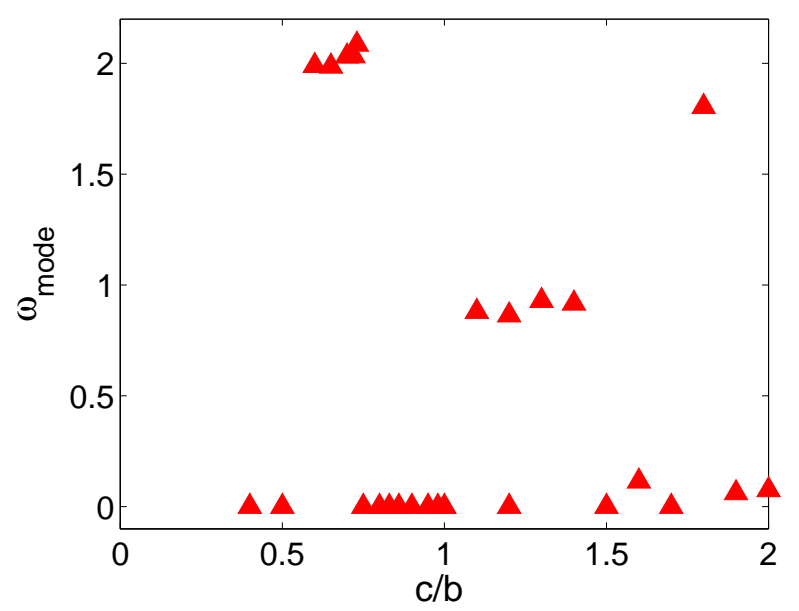

(b)

Figure 6: Influence of the length of the ellipsoid along the rotation axis on the mode selection of the tidal instability $(E=1 / 688, \varepsilon=0.317)$. (a) Variation of the growth rate. (b) Variation of the main frequency of the selected mode determined by Fourier analysis of its saturation state. 


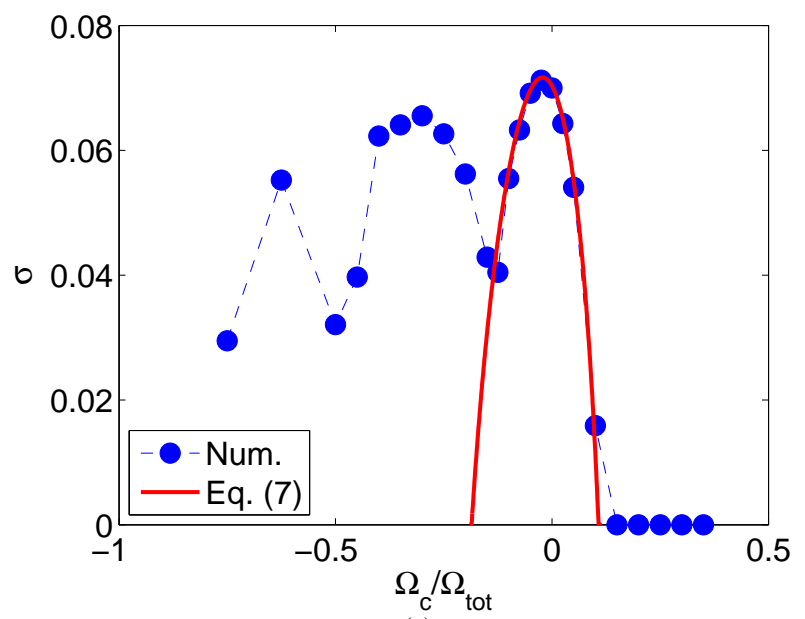

(a)

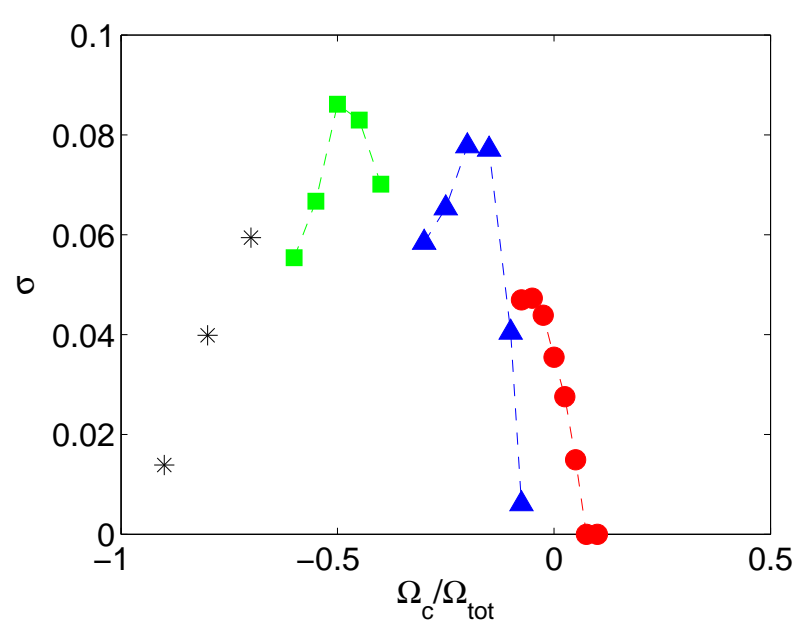

(b)

Figure 7: Evolution of the growth rate in the presence of a background rotation for a fixed ellipticity $\varepsilon=0.317$ and a fixed value of the total Ekman number $E_{\text {tot }}=\frac{v}{\Omega_{\text {tot }} R_{e q}^{2}}=10^{-3}$, where $\Omega_{\text {tot }}$ takes into account the background rotation and the fluid rotation, i.e. $\Omega_{\text {tot }}=\Omega_{c}+\Omega$. $(a) c=(a+b) / 2$, i.e. $c / b=0.86$ : the spin-over mode is then excited in the absence of background rotation. Good agreement is found around this value with the analytical solution (7). Further decreasing $\Omega_{c}$, other modes with smaller wavelength along the rotation axis appear. $(b) c / b=0.65$ : the $(1,3)$ mode is then excited in the absence of background rotation. Other modes can be excited: • represent the (1,3) mode, $\mathbf{\Delta}$ the spin-over mode, $\mathbf{\square}$ represent the $(-1,1)$ mode with one wavelength along the axis of rotation, and $*$ are other modes. 


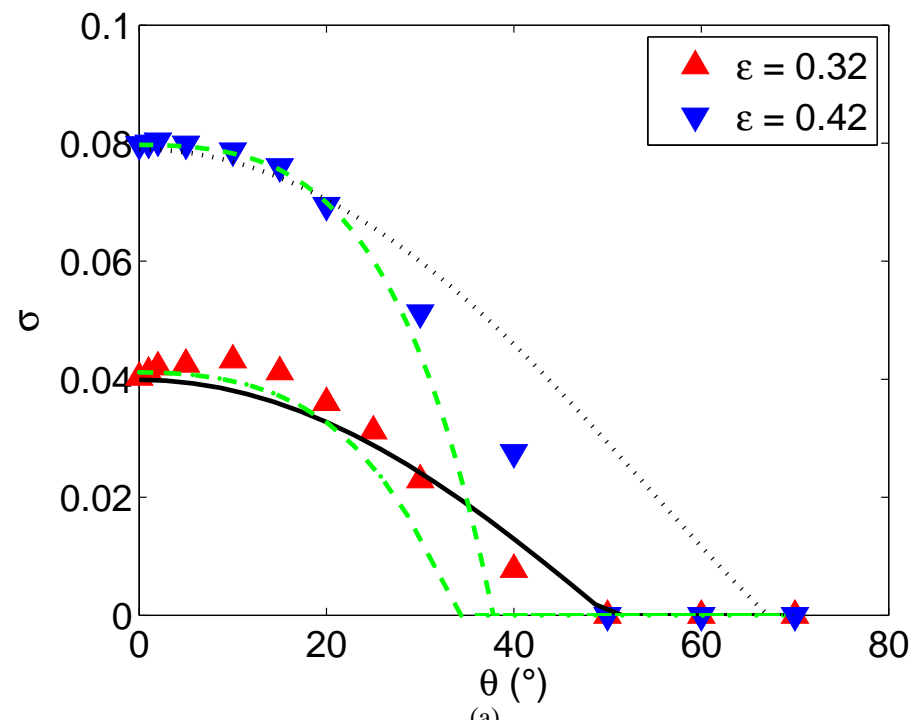

(a)

Figure 8: Evolution of the growth rate with the obliquity $\theta$ for $E=1 / 600$ (fixed angular rate) and two values of the ellipticity $\varepsilon=0.317$ and $\varepsilon=0.42$. The dashed dotted line and the dashed line correspond to expression (6), taking into account the values of apparent axes seen from the equatorial plane, whereas the dotted line and the continuous line correspond to expression (3), simply considering the apparent value of the ellipticity seen from the equatorial plane. The coefficient $K$ for viscous corrections is determined at $\theta=0^{\circ}$ and then kept constant. 


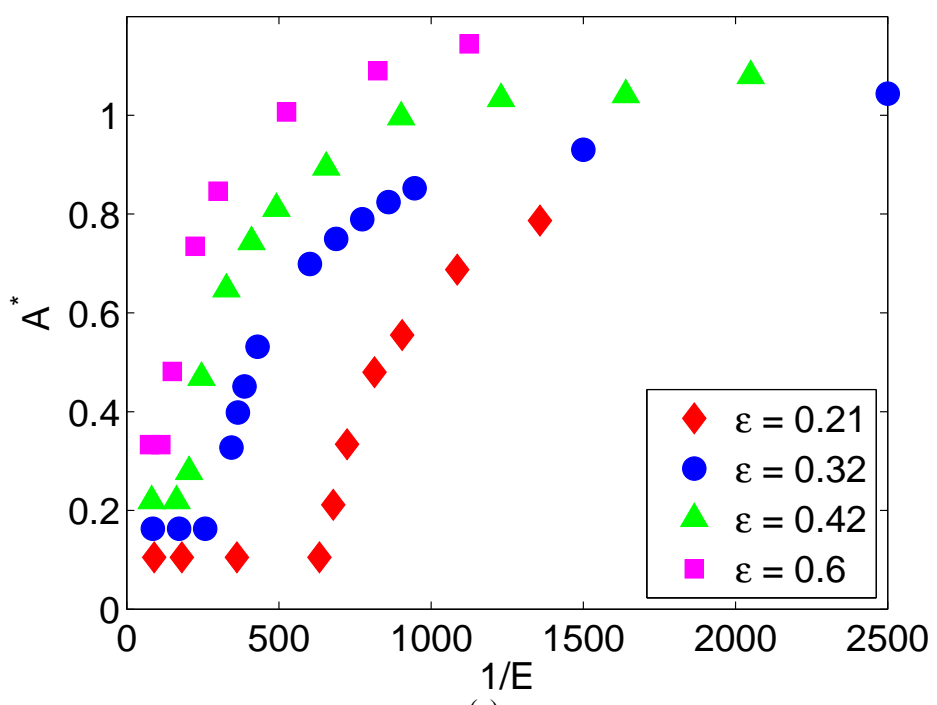

(a)

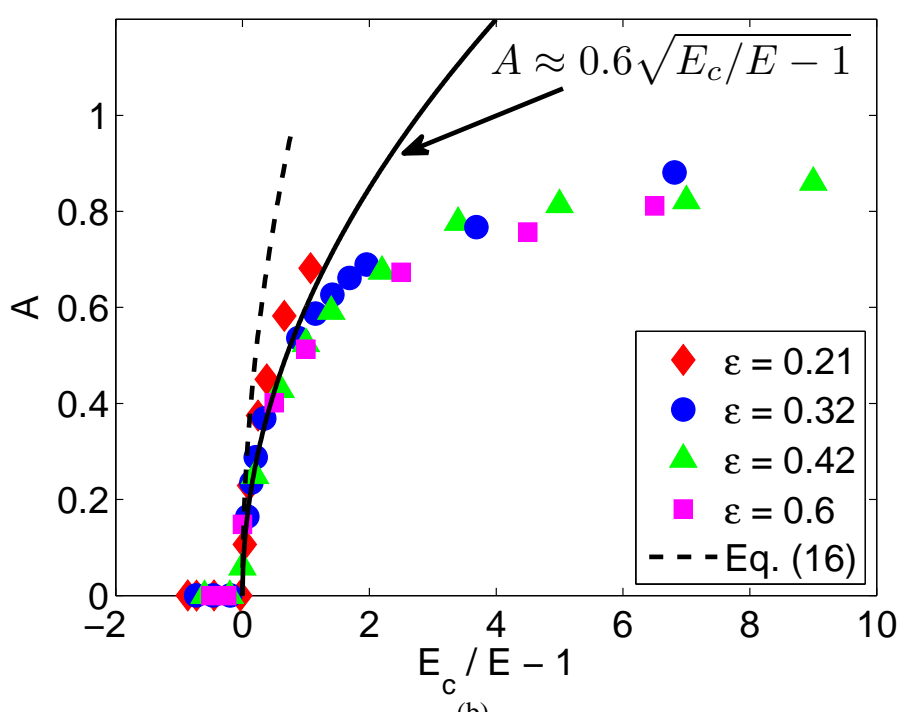

(b)

Figure 9: Variation of the saturation amplitude of the flow driven by the elliptical instability depending on the Ekman number, for various values of the ellipticity. (a) Maximum value of the difference between the actual velocity and the theoretical base flow (5). (b) Maximum amplitude of the tidal instability, corresponding to the previous values corrected to take into account recirculation cells: all curves then superimpose when computed as a function of $E_{c} / E-1$. 


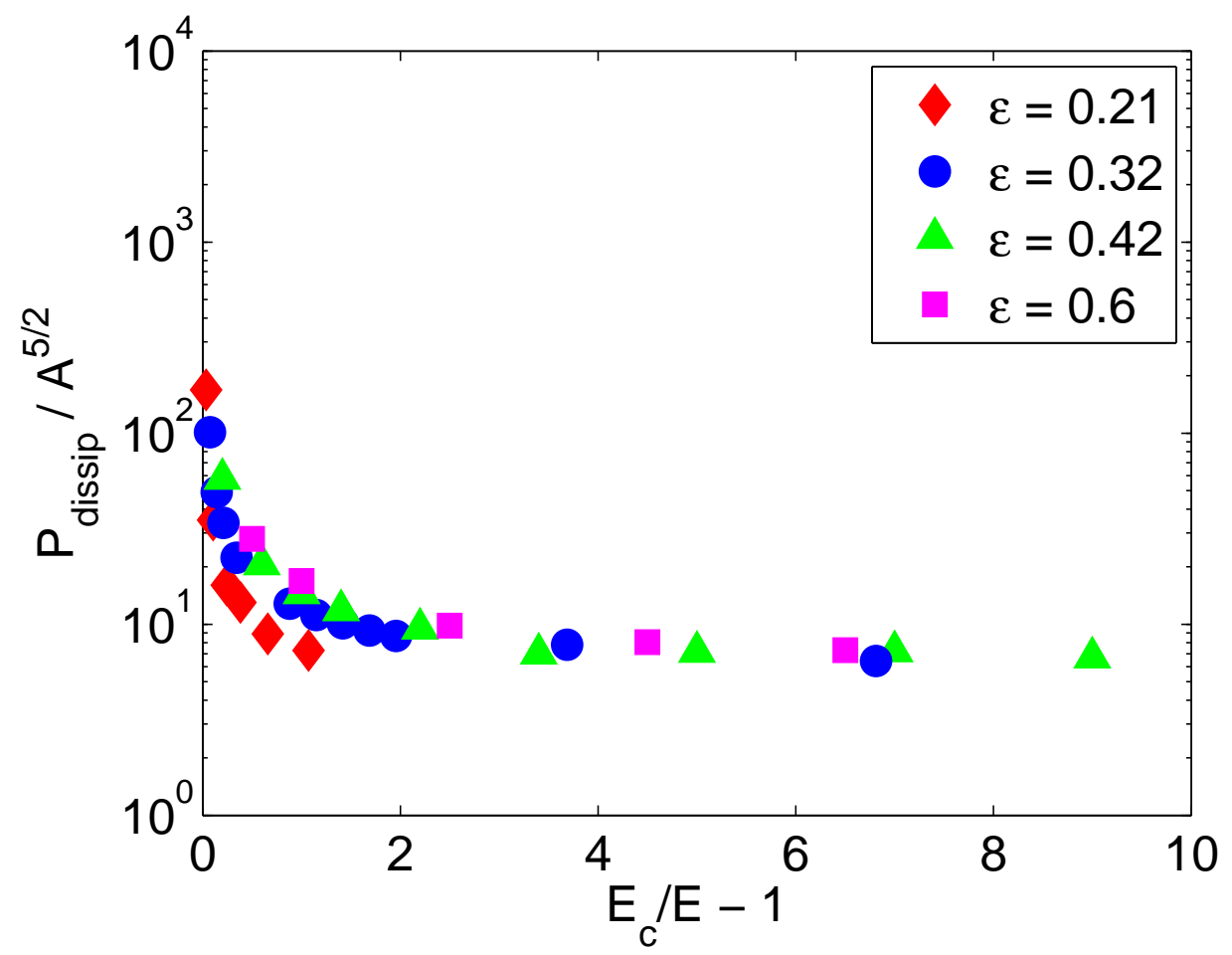

Figure 10: Viscous dissipation by the tidal instability, as a function of the distance from threshold. The results collapse on a generic law and seem to converge towards a saturation value far from threshold. 